\title{
Problemas de Relojes. Ejemplos Históricos y Consideraciones Didácticas
}

\section{Clock problems. Historical Examples and Didactical Considerations}

\author{
Vicente Meavilla Seguí* \\ Antonio M. Oller Marcén ${ }^{* *}$
}

\begin{abstract}
Resumen
Abordamos un problema matemático clásico: aquel en el que se trata de calcular el tiempo que debe transcurrir, a partir de una hora determinada, para que las manecillas de un reloj ocupen una posición concreta. En particular, nos centramos en el caso en que la disposición requerida es que las agujas estén superpuestas. En este artículo, presentamos diversos ejemplos extraídos de textos clásicos y del siglo XIX que ilustran distintos contextos en los que se presenta el problema, así como diferentes métodos de resolución. Además, como consecuencia de dicho análisis, presentamos algunas consideraciones didácticas que pueden motivar el trabajo de estos materiales con profesorado en formación.
\end{abstract}

Palabras clave: Historia de las Matemáticas. Educación Matemática. Aritmética. Álgebra. Problemas de relojes.

\begin{abstract}
We approach a classical Mathematical problem: that of computing the time passed, from a given moment, until the hands of a clock reach certain position. In particular, we focus on the case when the required position is the superposition of both hands. In this paper, we present some examples from classic and nineteenth century texts presenting different contexts where the problem arises as well as different solving methods. In addition, and as a consequence of this analysis, we present some didactical considerations that motivate the use of these resources with prospective teachers.
\end{abstract}

Keywords: History of Mathematics. Mathematics Education. Arithmetic, Algebra. Clock problems.

\footnotetext{
* Doctor por la Universidad de Barcelona (UAB). Profesor del Departamento de Matemáticas de la Universidad de Zaragoza, Facultad de Ciencias Sociales y Humanas (UNIZAR), Teruel, Aragón, España. Dirección Postal: Ciudad Escolar s/n, C.P. 44003, Teruel, España. E-mail: meavilla@ unizar.es

*** Doctor por la Universidad de Valladolid (UVA). Profesor del Centro Universitario de la Defensa de Zaragoza (CUD-ZGZ), Zaragoza, Aragón, España. Dirección Postal: Ctra. de Huesca s/n, C.P. 50090, Zaragoza, España. E-mail: oller@unizar.es
} 


\section{Introducción}

En un libro de texto español actual (ARIAS; MAZA, 2011) encontramos el siguiente problema.

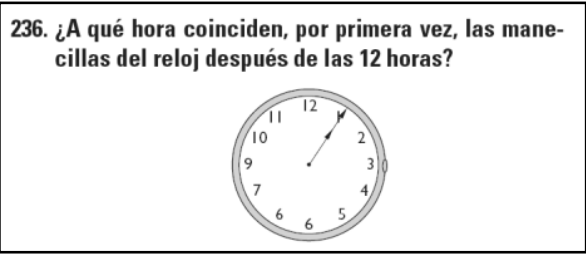

Figura 1 - Problema de superposición de agujas de un reloj.

Por su parte, en un texto bastante más antiguo (MOYA, 1867) se propone el siguiente enunciado.

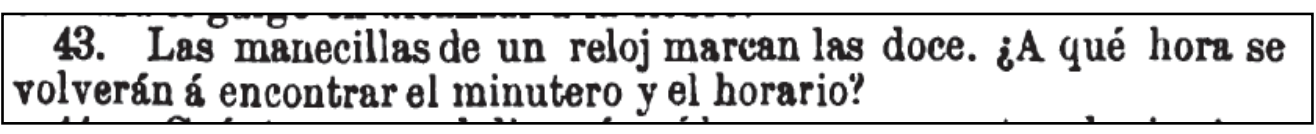

Figura 2 - El mismo problema de superposición de agujas, hace 150 años.

Pese a que entre ambos textos median prácticamente 150 años, se observa que el problema propuesto es idéntico. Sin embargo, el modo en que los autores conciben su resolución no es, en absoluto, el mismo.

Así, por una parte, los autores actuales proponen la siguiente solución al problema.

Sea $x$ el ángulo que recorre la aguja minutera.
$12(x-30)=x \Rightarrow x=32,73^{\circ}$
Se encontrarán cuando la aguja minutera haya recorrido un
ángulo de $32,73^{\circ}$, es decir, $32,73^{\circ}: 30=1,09 \mathrm{~h}=1$ hora 5
minutos 24 segundos.

Figura 3 - Solución algebraica actual al problema de superposición de las agujas de un reloj.

Sin embargo, la obra de Ambrosio Moya se titula Lecciones de Aritmética y el citado problema aparece bajo el epígrafe Ejercicios sobre el cálculo de los números concretos. En consecuencia, la solución esperada por este autor debía ser puramente aritmética y estaría bastante alejada de la anterior.

La existencia de familias de problemas que aparecen repetidamente en la Historia de las Matemáticas, en textos de diferentes épocas y culturas, resolviéndose de diversas formas, 
es un fenómeno común e interesante. Por ejemplo, en un reciente artículo, Meavilla y Oller (2014) analizan detalladamente el caso de los llamados problemas de grifos.

En este trabajo nos centramos en un tipo de problemas que denominamos de relojes. En ellos se trata de calcular el tiempo que debe transcurrir, a partir de una hora determinada, para que las manecillas de un reloj ocupen una posición concreta. Las disposiciones que suelen requerirse con una mayor frecuencia son:

1. las manecillas están superpuestas (conjunción),

2. las manecillas forman ángulo recto (cuadratura),

3. las manecillas forman un ángulo llano (oposición).

En nuestro estudio presentamos únicamente problemas de conjunción. Evidentemente, esto no supone restricción alguna puesto que las ideas implicadas en las resoluciones de cada uno de ellos son idénticas.

\section{Problemas de relojes sin relojes}

Los primeros instrumentos mecánicos dedicados a la medida del tiempo (y de otros fenómenos astronómicos) de los que se tiene noticia surgieron en la China del siglo VIII. Estos ingenios mecánicos chinos, que se impulsaban con agua o mercurio, alcanzaron su esplendor en torno a los siglos X-XI (NEEDHAM; LING; DE SOLLA PRICE, 1960). Sin embargo, fue en la Europa de finales del siglo XIII (CROSBY, 1998) cuando se inició el desarrollo de los relojes mecánicos modernos no impulsados por agua.

Inicialmente, los relojes no mostraban la hora (carecían de esfera) y simplemente hacían sonar campanas. Con la invención de la esfera se introdujo una manecilla que señalaba la hora, pero hasta la introducción del péndulo como elemento regulador del mecanismo, en el siglo XVII, no se logró la precisión suficiente como para incluir una manecilla que señalase los minutos.

Cabría esperar, por tanto, que no aparecieran problemas similares al de la Figura 1 en textos anteriores a esa época. Sin embargo, una búsqueda en textos anteriores al siglo XVII demuestra que es posible encontrar problemas equivalentes al inicial, pero propuestos en contextos distintos a los del movimiento de las manecillas en la esfera de un reloj. Incluso, en uno de los casos vemos que, sorprendentemente, los datos son exactamente los mismos que en el caso del reloj. 


\subsection{Un problema de Luca Pacioli}

El ejemplo más antiguo que vamos a presentar proviene de la Summa de Arithmetica Geometria Proportioni et Proportionalità (PACIOLI, 1494). En concreto, se trata del problema número 80, propuesto en el Tractatus geometrie. Distinctio octaua (PACIOLI, 1494, p. 64). El franciscano italiano Luca Pacioli plantea la siguiente cuestión equivalente al problema de superposición de las dos agujas de un reloj:

Son dos circunferencias, una mayor que la otra, una dentro de la otra y con el mismo centro. Un hombre camina sobre la circunferencia grande y la circunda en 12 días. Y sobre la circunferencia pequeña camina otro hombre y la circunda en 24 horas. $\mathrm{Si}$ estos dos hombres salen a la vez, en el mismo sentido y desde el mismo lugar, pregunto: ¿en cuántos días volverán a estar juntos?

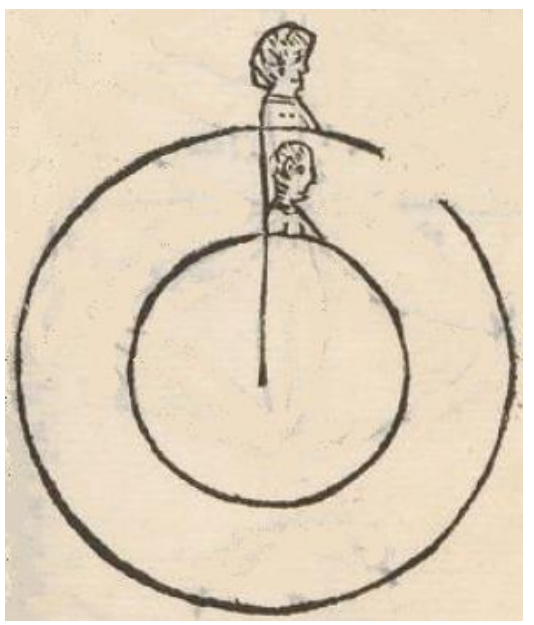

Figura 4 - Figura original que acompaña al problema de Pacioli

Llama la atención que, pese a encontrarnos en un contexto ajeno al funcionamiento de un reloj y pese a que en el momento en que Pacioli compuso su Summa los relojes aún no disponían de dos manecillas, la razón entre las velocidades angulares de los hombres es la misma que la que existe entre las del minutero y el horario de un reloj (12:1).

La solución propuesta por Pacioli está escrita en lenguaje retórico y sin apenas símbolos algebraicos. Tan solo utiliza las expresiones co para referirse a la incógnita y $p$ para denotar el símbolo +. A continuación, presentamos brevemente la solución de Pacioli adaptada al lenguaje actual. S

Supongamos que el encuentro tiene lugar al cabo de $1+x$ días. Si el hombre más lento da una vuelta completa en 12 días, entonces en $1+x$ días dará $1 / 12+x / 12$ vueltas. Por su parte, si el hombre más rápido da una vuelta completa en 1 día, entonces en $1+x$ días dará 1 $+x$ vueltas. En consecuencia se tiene que $x / 12+1 / 12=x$. Basta resolver esta ecuación para 
obtener que $x=1 / 11$ días y, por tanto, ambos hombres estarán alineados con el centro por primera vez después de $1+1 / 11=12 / 11$ días.

Esta solución merece un comentario, en especial en lo relativo a la elección de la incógnita (ver Figura 5). Puede resultar extraño no tomar como incógnita el número de días al cabo de los cuales se producirá el encuentro. Sin embargo, por las condiciones del problema es evidente que ha de pasar un día completo (y menos de dos) antes de que los hombres se encuentren. Equivalentemente, el hombre más lento habrá recorrido menos de una vuelta en ese tiempo y el más rápido más de una vuelta, pero menos de dos. En este escenario, Pacioli toma para la incógnita el valor de $1+x$ días. Esta elección facilita el planteamiento de la ecuación puesto que el ángulo recorrido por el hombre más lento (menos de una vuelta) coincide con la parte del ángulo recorrido por el hombre más rápido (más de una vuelta, pero menos de dos) que excede a una vuelta.

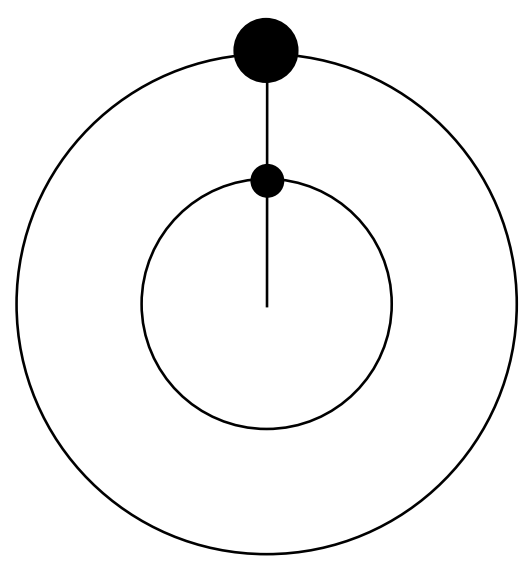

Posición inicial

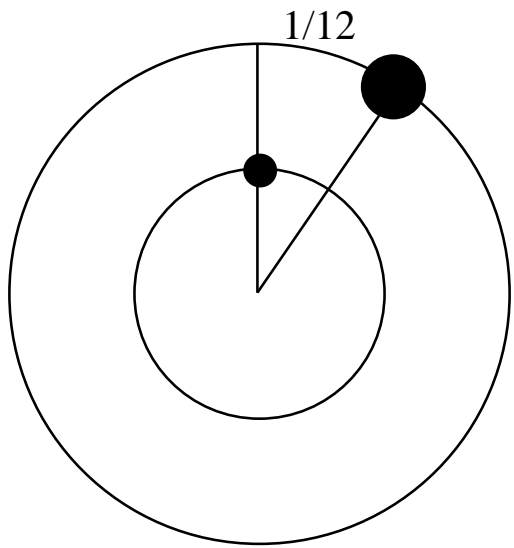

Posición al cabo de un día

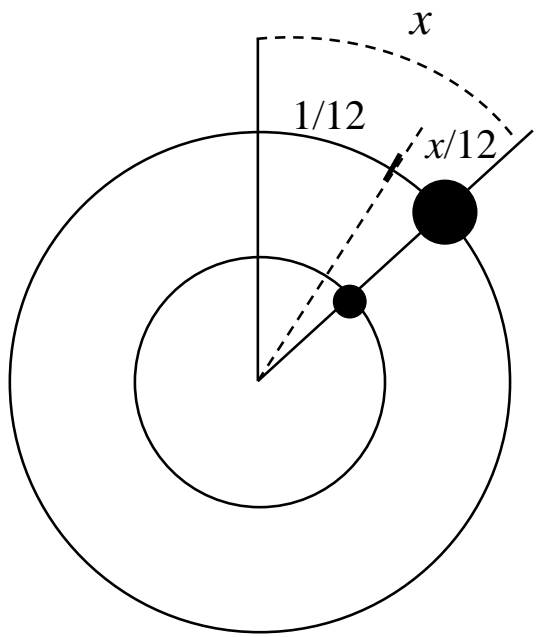

Posición al cabo de $1+x$ días

Figura 5 - Esquema de la solución de Pacioli.

\subsection{Un problema del siglo XVI}

En la Pratica mercantíuol del mallorquín Joan Ventallol encontramos el siguiente problema de persecución que claramente pertenece a la misma familia que los problemas de relojes (VENTALLOL, 1521, fols. XCVr-XCVv):

Dos hombres corren alrededor de una ciudad redonda y amuralla. Los dos empiezan a correr al mismo tiempo y desde el mismo lugar [y en el mismo sentido]. Uno tarda 4 horas en dar una vuelta completa y el otro necesita $5 \frac{1}{2}$ horas. Corren hasta que el corredor más rápido alcanza al otro [...]

Pregunto: ¿en cuántas horas lo conseguirá? 
El contexto es similar al del problema planteado por Pacioli en la Summa; es decir, hombres que recorren un mismo itinerario circular a distintas velocidades. Ventallol, sin embargo, trata de presentar el problema en una situación más realista en la que los hombres rodean una ciudad.

La solución del mallorquín es radicalmente diferente de la dada por Pacioli:

\begin{abstract}
Esta es la regla:
Multiplica 4 por $51 / 2$ [debe decir 11], encontrarás 44 [...] Ahora divide 44 por 4 y vendrán 11. Entonces, el de las 4 horas habrá rodeado 11 veces la ciudad en 44 horas. Después, divide 44 por $51 / 2$ y vendrán 8 . Y tantas veces habrá rodeado la ciudad el de $51 / 2$ en 44 horas. Mira ahora cuánto hay de 8 a 11 y encontrarás 3 . Y en tanto aventaja el uno al otro. Divide 44 por 3 y vendrán 14 2/3. En tantas horas lo habrá conseguido.
\end{abstract}

Esta resolución es de carácter puramente aritmético. La idea principal supone considerar un número arbitrario de horas (para facilitar los cálculos se toma un valor de forma que al dividirlo por 4 y por 11/2 ambos resultados sean enteros) y calcular cuántas vueltas de ventaja ha obtenido el rápido sobre el lento en ese tiempo. Conocido ese dato es fácil calcular el tiempo en el que el rápido aventaja al lento exactamente en una vuelta que, evidentemente, es el tiempo que tarda en alcanzarlo.

Este esquema de resolución es muy común en problemas de persecuciones y está íntimamente relacionado con la Regla de Falsa Posición (MEAVILLA, 2005).

\title{
2.3 Un problema del siglo XVII
}

El último ejemplo de problema que vamos a presentar proviene de la Arithmetica practica de Gerónimo Cortés. En dicho texto (CORTÉS, 1659, p. 456) encontramos un problema similar a los anteriores pero en un contexto relacionado con cálculos astronómicos ${ }^{1}$.

D. Si oy se hallassen dos estrellas, o planetas juntos, y en conjuncion, como sabriamos por Arithmetica sin ser Astronomos en quanto tiempo se tornarian a hallar juntos, como sucede en el presente año, entre Iupiter y Saturno, que se hallan juntos la víspera de Navidad, el qual ajuntamiento llaman los Astronomos, conjuncion magna, por los grandes, y terribles effectos que suele causar, segun ellos dizen, y la experiencia lo demuestra.

M. Essa demanda bien la pudieras aver dexado para los Astronomos pues a ellos toca; pero toda via quiero darte contento; y advierte, que primero se ha de saber quanto tiempo tarda cada estrella, o planeta en darla buelta a todo su orbe. Y pues has hecho memoria de la magna conjuncion de Iupiter, y Saturno, propo(n)gamos el exemplo dellos. Y sepas que Iupiter tarda en dar la buelta à su orbe doze años, y Saturno al suyo tarda treynta años, segun parecer de Cardano, porque unos escribe(n) que tardan mas, y otros menos; y tomando el parecer de Cardano, digo,

\footnotetext{
${ }^{1}$ La primera edición es de 1604. En el texto original, $\mathrm{D}=$ discípulo, $\mathrm{M}=$ maestro.
} 
que multipliques los 12 años de Iupiter por los 30 de Saturno, y montaran 360 años (i), que partidos por 18 que es la differencia que hay de 12 à 30 saldran 20 años, y acabo de tantos años se hallaran ju(n)tos, y en conjunción los dichos planetas.

En este caso, el contexto en que se plantea el problema es mucho más interesante que en los ejemplos anteriores. De hecho, se trata de un fenómeno real. Sin embargo, dejando de lado este interés, la solución dada por Cortés es idéntica a la presentada por Ventallol y es, por tanto, puramente aritmética.

\section{Relojes en el siglo XIX}

Respecto del siglo XIX, José Manuel Sánchez Ron señaló que (SÁNCHEZ, 1992, p. 58): “el ámbito en el que se movieron los físicos y matemáticos españoles de aquella centuria fue, con muy pocas excepciones, el de la enseñanza”. Por su parte (GÓMEZ, 2011, p. 13) apunta la "popularización sin precedentes" que tuvieron los libros de texto de matemáticas en esa época.

Ante este auge de los libros de texto, no es de extrañar que encontremos entre ellos múltiples ejemplos de problemas de relojes. En esta sección vamos a presentar algunos ejemplos propuestos por autores del XIX.

El gijonés Acisclo Fernández Vallín y Bustillo propone en sus Elementos de Matemáticas dos soluciones algebraicas distintas al problema que hemos presentado en la introducción (FERNÁNDEZ-VALLÍN, 1857, p. 87):

Siendo en un reloj las 12 en punto, y estando por consiguiente el minutero sobre el horario, ¿qué hora será cuando el minutero vuelva a colocarse sobre el horario?

Tomando por unidad de camino la dozava parte de la circunferencia del reloj, y llamando $x$ el camino que anda el horario hasta que le alcance el minutero después de la una, este andará evidentemente $1+x$; y como en tiempo igual el minutero camina doce veces más que el horario, tendremos $1+x=12 x$, de donde resulta $x=$ $1 / 11$ de hora, es decir que la hora pedida será la 1 y $1 / 11$ o bien la 1 y 5 y $5 / 11$ minutos. Al cabo de igual tiempo o sea a las 2 y 2/11, 3 y 3/11 etc., se encontrarán de nuevo, repitiéndose lo mismo hasta las 12 en punto.

De otro modo: tomando la hora por unidad y llamando $x$

el tiempo que se busca tendremos $x=1+x / 12$ y $x=1$ hora 5 minutos $273 / 11$ segundos.

Este ejemplo es interesante, por cuanto ilustra las diferentes elecciones que pueden hacerse al fijar la variable incógnita. La primera de las soluciones presentadas por FernándezVallín coincide, en esencia, con el enfoque de Pacioli (tomar como incógnita el espacio recorrido hasta encontrarse) y conduce exactamente a la misma ecuación. La segunda 
alternativa (tomar como incógnita directamente el tiempo pedido) proporciona una ecuación diferente aunque obviamente equivalente.

Antonio Terry y Rivas, gaditano y Contralmirante de la Armada, en sus Ejercicios de Álgebra (TERRY, 1885a, p. 96) nos presenta un problema similar al original: "Siendo en un reloj las 12 en punto, y estando por consiguiente el minutero sobre el horario, ¿qué hora será cuando el minutero vuelva colocarse sobre el horario entre las 2 y las 3 ?".

La solución presentada por Terry (1885b, p. 91) coincide, esencialmente, con la segunda de las presentadas por Fernández-Vallín, con las variaciones que causa la diferencia en el enunciado:

Sea $x$ el número de minutos pedido después de las 2, en $x$ minutos el minutero andará $x$ divisiones de la circunferencia del reloj; y como éste anda 12 veces más que el horario, éste no andará más que $x / 12$ divisiones en $x$ minutos $x=x / 12+10$; A las 2 horas 10 minutos 10/11

Si comparamos las soluciones de Terry y de Fernández-Vallín, observamos la diferente unidad de medida utilizada por ambos para subdividir la circunferencia. Mientras Fernández-Vallín considera doce partes (correspondientes a las horas), Terry considera la subdivisión en minutos (es decir, 60 partes). Curiosamente, en la solución actual presentada en la Figura 3 la subdivisión se hace en grados (es decir, en 360 partes). Aunque, evidentemente, todas las opciones son igualmente válidas es importante hacer explícita la elección a la hora de plantear la ecuación correspondiente. Fernández-Vallín y Terry lo hacen, no así sus colegas contemporáneos.

Cerramos esta sección presentando una curiosa solución de carácter aritmético que hace uso de una idea muy interesante y que no proviene de la consideración de un problema de móviles de la manera usual. En concreto, aparece en el texto Lecciones de Aritmética del francés P.L. Cirodde, traducido al castellano en 1867, por Francisco Zoleo. El enunciado y su solución discurren del siguiente modo (CIRODDE, 1867, p. 121):

Un reloj señala las 12 , de modo que la manecilla de los minutos coincide con la de las horas: ¿a qué hora volverán a coincidir?

Es claro que si aumentan o disminuyen en una misma cantidad las velocidades de ambos móviles, sus movimientos relativos no se alterarán [...] Luego, si se disminuyen las velocidades de nuestras dos manecillas en la misma de la de las horas, lo que reducirá a esta al reposo, siempre la de los minutos necesitará el mismo tiempo para alcanzar a la de las horas. Ahora bien, esta última recorre en una hora 5 divisiones del cuadrante, mientras que la otra recorre 60 de dichas divisiones; la cuestión se reducirá, pues a esta: El minutero señala las doce; el recorre 55 divisiones en una hora: ¿cuánto tiempo invertirá para volver a su punto de partida, es decir, para recorrer 60 divisiones?

Resultado: 1 h 5, 5/1 
Esta solución, que evita el uso del Álgebra, vuelve a enfocar el problema desde el punto de vista de los móviles. Sin embargo, la idea es esencialmente distinta a la presentada en los apartados anteriores, puesto que consiste en plantear un problema equivalente, haciendo uso del principio de relatividad, en el que una de las manecillas está en reposo. Se trata de un razonamiento bastante sofisticado que, sin embargo, se puede aplicar a cualquier problema de persecuciones.

\section{Algunas consideraciones didácticas}

En el discurso anterior fueron traídos diferentes ejemplos históricos del modo en que se ha presentado y resuelto el problema de la conjunción de las manecillas de un reloj. El uso de fuentes originales es un importante recurso a la hora de trabajar en el aula de matemáticas (JAHNKE et al., 2000). En particular, este uso puede resultar de especial interés en el trabajo con profesorado en formación (SMESTAD, 2011). En esta sección presentamos algunas cuestiones que surgen de forma natural a partir del análisis de los textos presentados y que ilustran temas interesantes que podrían tratarse en un hipotético trabajo de aula.

\subsection{Respecto al carácter algebraico o aritmético del problema}

El problema que nos ocupa se puede resolver tanto aritmética como algebraicamente. Hemos visto, de hecho, que ambos tipos de resoluciones conviven desde las primeras apariciones de este tipo de problema.

Cerdán (2008, p. 48) indica que "hay un tipo de problemas verbales que parecen obligar al uso del razonamiento algebraico para poder resolverlos”. Bajo este punto de vista, y a la luz de los ejemplos mostrados, no parece que estemos ante un problema de este tipo. De hecho, no apreciamos obligación alguna que nos decida a optar por razonamientos algebraicos.

En cuanto a cuestión sobre cuál de las dos opciones es mejor, su respuesta (si existe) es puramente subjetiva. Las soluciones aritméticas necesitan que el resolutor maneje con gran profundidad los significados de los números racionales y de las operaciones con ellos. El uso del álgebra, con toda su potencia, puede oscurecer la comprensión de lo que sucede en el 
problema y su utilización requeriría, en nuestra opinión, de un conocimiento previo de la solución aritmética.

\subsection{Unidades de medida}

En los ejemplos propuestos hemos encontrado hasta tres modos de subdividir la circunferencia del reloj; es decir, hasta tres modos de medir el desplazamiento de las agujas. La diferencia radica, justamente, en la unidad elegida. Las tres unidades observadas han sido:

a. El grado sexagesimal, en el texto de Arias y Maza (2011).

b. El desplazamiento del minutero en un minuto (1/60 de circunferencia; es decir, 6 grados), en el texto de Terry (1885b).

c. El desplazamiento del horario en una hora (1/12 de circunferencia; es decir, 30 grados), en el texto de Fernández-Vallín (1857).

Esta elección es muy relevante, pues en función de la opción elegida el espacio recorrido, por ejemplo, por el horario en una hora es de 30, 5 ó 1 unidades, respectivamente. El manejo adecuado de la unidad de medida es especialmente importante en el trabajo con el número racional (ESCOLANO, 2007).

Además, en el caso que nos ocupa, también es importante esta elección en el proceso de resolución algebraica del problema, puesto que según la unidad elegida la ecuación a resolver será distinta. En concreto, si $x$ es el espacio recorrido por el horario hasta ser alcanzado:

a. Si la unidad es el grado, tenemos $30+x=12 x$.

b. Si la unidad es de 6 grados, tenemos $5+x=12 x$.

c. Si la unidad es de 30 grados, tenemos $1+x=12 x$.

Las tres ecuaciones anteriores tienen soluciones diferentes si se consideran en abstracto. Sin embargo, si se consideran en el contexto del problema y se entiende que los números implicados son cantidades de magnitud medidas con una unidad concreta, todas ellas llevan a la misma solución:

a. $x=30 / 11$ de grado.

b. $x=5 / 11$ de 6 grados.

c. $x=1 / 11$ de 30 grados. 
Así, pues, el uso de unidades de medida trasciende la aritmética y tiene su importancia también en el plano algebraico. Estas consideraciones pueden ayudar a comprender algunos errores cometidos por los alumnos en el proceso de transición de la Aritmética al Álgebra (SOCAS, 2011).

\subsection{Una propuesta de actividad}

Como ya hemos mencionado, el uso de fuentes originales puede resultar de especial interés en el trabajo con profesorado en formación. Vamos a cerrar este trabajo presentando una posible secuencia de actividades con la que trabajar con futuros docentes de Secundaria:

1. Busca información sobre Luca Pacioli. ¿Quién fue? ¿cuándo y dónde vivió? ¿qué obras escribió?

2. En la obra Summa de Arithmetica Geometria Proportioni et Proportionalità de Luca Pacioli (1494, p. 64) encontramos el siguiente problema:

Son dos circunferencias, una mayor que la otra, una dentro de la otra y con el mismo centro. Un hombre camina sobre la circunferencia grande y la circunda en 12 días. Y sobre la circunferencia pequeña camina otro hombre y la circunda en 24 horas. $\mathrm{Si}$ estos dos hombres salen a la vez, en el mismo sentido y desde el mismo lugar, pregunto: ¿en cuántos días volverán a estar juntos?

Resuélvelo, utilizando ecuaciones, indicando paso a paso cómo lo has hecho.

3. Cuando lo resolvió, hace más de 500 años, Pacioli plateó la ecuación $x / 12+1 / 12=x$. ¿Coincide con la que tú has obtenido? ¿Qué has tomado como incógnita? ¿Qué tomó Pacioli como incógnita para llegar a esa ecuación?

4. Resuelve el problema de nuevo, pero esta vez sin recurrir al uso de ecuaciones. ¿Cómo lo has hecho?

5. En el siglo XVI, Joan Ventallol, propuso una regla para resolver este tipo de problemas. En nuestro caso sería de la siguiente manera (expresado en lenguaje moderno):

Considera el tiempo que tarda cada hombre en dar una vuelta completa: 12 días y 1 día. Multiplica esos valores y obtendrás 12. Divide este 12 entre los tiempos anteriores (12 y 1) y obtendrás 1 y 12, respectivamente. Resta 12 menos 1, sale 11. Divide 12 entre 11. El resultado, $12 / 11$, es el tiempo que tardan en volver a juntarse.

Interpreta las operaciones realizadas por Ventallol y explica el método de resolución.

6. A partir del apartado anterior busca una fórmula que permita resolver el siguiente problema general: 
Son dos circunferencias, una mayor que la otra, una dentro de la otra y con el mismo centro. Un hombre camina sobre la circunferencia grande y la circunda en $a$ días. Y sobre la circunferencia pequeña camina otro hombre y la circunda en $b$ días. Si estos dos hombres salen a la vez, en el mismo sentido y desde el mismo lugar, pregunto: ¿en cuántos días volverán a estar juntos?

\section{Referencias}

ARIAS, J.M; MAZA, I. Matemáticas ESO 3. Madrid: Bruño, 2011.

CERDÁN, F. Estudios sobre la familia de problemas aritmético-algebraicos. Valencia: Servei de Publicacions, 2008.

CIRODDE, P. L. Lecciones de Aritmética. Madrid: Carlos Bailly-Bailliere, 1867.

CORTÉS, G. Arithmetica practica. Valencia: Iuan Lorenzo Cabrera, 1659.

CROSBY, A.W. La Medida de la realidad. Barcelona: Crítica, 1998.

ESCOLANO, R. Enseñanza del número racional positivo en Educación Primaria: un estudio desde los modelos de medida y cociente. 2007. 2v. Tese (Doutorado em Matemática) - Faculdade de Ciencias, Universidad de Zaragoza, Zaragoza, 2009.

FERNÁNDEZ-VALLÍN, A. Elementos de Matemáticas. 6 ed. Madrid: Imprenta del Colegio de Sordo-mudos y de Ciegos, 1857.

GÓMEZ, B. Marco preliminar para contextualizar la investigación en historia y educación matemática. Epsilon, Sevilla, v. 28, n. 77, p. 9-22, jan./abr. 2011.

JAHNKE, H.N.; ARCAVI, A.; BARBIN, E.; BEKKEN, O.; FURINGHETTI, F.; EL IDRISSI, A.; SILVA, C.M.; WEEKS, CH. The use of original sources in the mathematics classroom. In: FAUVEL, J.; VAN MAANEN, J. (Ed.). History in mathematics education: the ICMI study. Dordrecht: Kluwer, 2000. p. 291-328.

MEAVILLA, V. La historia de las matemáticas y la resolución no algebraica de problemas: una propuesta didáctica. In CARRASQUER, J. (Ed.). La Historia de la Ciencia como recurso de aula y de Investigación Didáctica. Teruel: Universidad de Zaragoza, 2005. p. 99-120.

MEAVILLA, V.; OLLER, A. M. Entre la Aritmética y el Álgebra. Un análisis histórico de los "problemas de grifos". Revista Educación Matemática, México, v. 26, n. 1, p. 9-32, abr. 2014.

MOYA, A. Lecciones de Aritmética. Madrid: Imprenta de Segundo Martínez, 1867.

NEEDHAM, J.; LING, W.; DE SOLLA PRICE, D.J. Heavenly Clockwork: The Great Astronomical Clocks of Medieval China. Cambridge: Cambridge University Press, 1960.

PACIOLI, L. Summa de Arithmetica Geometria Proportioni et Proportionalità. Venezia: Paganini, 1494.

SÁNCHEZ, J. M. Las ciencias Físico-Matemáticas en la España del siglo XIX. In: LÓPEZ PIÑERO, J.M. (Ed.). La Ciencia en la España del siglo XIX. Madrid: Marcial Pons, 1992. p. 51-84. 
SMESTAD, B. History of Mathematics for Primary School Teacher Education Or: Can You Do Something Even if You can't Do Much? In: KATZ, V.; TZANAKIS, C. (Ed.). Recent developments on introducing a historical dimension in Mathematics Education. Washington: MAA, 2011.p. 201-210.

SOCAS, M. La enseñanza del álgebra en la Educación Obligatoria. Aportes desde la investigación. Números, La Laguna, v. 77, n. 1, p. 5-34, jul. 2011.

TERRY, A. Ejercicios de Álgebra parte originales y parte escogidos de los principales autores que tratan de la materia (Primera Parte). Madrid: Imprenta de la viuda e hijos de Abienzo, 1885a.

TERRY, A. Soluciones de los ejercicios de Álgebra (Segunda Parte). Madrid: Imprenta de la viuda e hijos de Abienzo, $1885 b$.

VENTALLOL, J. Pratica mercantíuol. Lyo: Joan de la Place, 1521.

Submetido em Abril de 2014. Aprovado em Junho de 2014. 